TITLE:

\title{
PRELIMINARY LIST OF
}

OCTOCORALS OF SAGAMI BAY

DEPOSITED IN THE BIOLOGICAL LABORATORY OF THE IMPERIAL HOUSEHOLD

\author{
$\operatorname{AUTHOR}(\mathrm{S})$ : \\ Utinomi, Huzio
}

CITATION:

Utinomi, Huzio. PRELIMINARY LIST OF OCTOCORALS OF SAGAMI BAY DEPOSITED IN THE BIOLOGICAL LABORATORY OF THE IMPERIAL HOUSEHOLD. PUBLICATIONS OF THE SETO MARINE BIOLOGICAL LABORATORY 1962, 10(1): 105-108

\section{ISSUE DATE:}

1962-07-30

URL:

http://hdl.handle.net/2433/175297

RIGHT: 


\title{
PRELIMINARY LIST OF OCTOCORALS OF SAGAMI BAY DEPOSITED IN THE BIOLOGICAL LABORATORY OF THE IMPERIAL HOUSEHOLD ${ }^{1)}$
}

\author{
Huzio UTINOMI \\ Seto Marine Biological Laboratory \\ Sirahama, Wakayama-ken \\ STOLONIFERA \\ Family Cornulariidae
}

(Coel. Sp. No.)

1. Cornularia sagamiensis UTINOMI (n. sp. ${ }^{2}$ ) $342,343,364$, $497,613,614$, 622.

2. Cornularia aggregata UTiNomi (n. sp.) $390,539,633$.

Family Clavulariidae

3. Clavularia spongicola UTINOMI (n. sp.) $310,314,365$. $442,450,466$, $520,547,583$, $589,629,631$, 645 (?).

4. Clavularia multispiculosa UTINOMI (n. sp.) 463.

5. Clavularia mikado UтіNomi (n. sp.) $452,660,661$.

6. Clavularia eburnea KÜKENTHAL? 737.

\section{TELESTACEA}

Family Telestidae

7. Telesto tubulosa Kinoshita $543,777,791$.

8. Telesto sagamiana KinoshITA? 140.

1) Contributions from the Seto Marine Biological Laboratory, No. 382.

2) "n. sp." in this list indicates new species previously described or will be described later, basing upon materials of this collection.

Publ. Seto Mar. Biol. Lab., X (1), 1962. (Article 6) 
Family Pseudocladoconidae

9. Pseudocladoconus hicksoni VeRsLUYS $575,681$.

\section{ALCYONACEA}

Family Alcyoniidae

10. Bellonella rubra BRUNDIN $46,51,570$, 662,710 .

11. Bellonella grandiflora (KÜKENTHAL) 298.

12. Bellonella unicolor (KÜKENTHAL) $620,623,713$.

13. Bellonella rigida PÜTTER 299, 368, 372, 374,609 .

14. Bellonella pellucida (KÜKenthaL) 28.

15. Bellonella albiflora Uтімomr (n. sp.) 632.

16. Bellonella dofleini (KÜKENTHAL) $654,701,717$.

17. Bellonella macrospina (KüKENTHAL) $433,540,558$, $653,755$.

18. Alcyonium gracillimum KÜKENTHAL $358,565,655$, 726 (?).

19. Minabea robusta UTINOMI (n. sp.) 50.

20. Cladiella digitulata (KLUNZINGER) $572,666,668$, $669,670,752$, 801.

\section{Family Nephtheidae}

21. Litophyton graeffei (KüKENTHAL)

248,249 .

22. Litophyton stella UтіNomi (n. sp.) ...?

23. Lemnalia laevis ThOMSON \& DEAN 473.

24. Daniela koreni voN $\mathrm{KoCH}$ $585,619,747$, $757,760,764$.

25. Duva bicolor (UTINOMI) 155.

26. Paraspongodes spiculosa (KÜKENTHAL) $359,598,621$, $634,635,746$, 778,790 .

27. Paraspongodes hirotai (UTINOMI) 67, 748 .

28. Dendronephthya (Dendronephthya) gigantea (VERRILI) ............. 126.

29. Dendronephthya (Dendronephthya) aurea UTINOMI. 774,776 .

30. Dendronephthya (Dendronephthya) albiflora U UімомI (n. sp.) 646,647 .

31. Dendronephthya (Roxasia) suensoni (HoLM) $477,478,488$. 
32. Dendronephthya (Roxasia) golgotha UTINOMI 475,534 .

33. Dendronephthya (Roxasia) japonica KÜKENTHAL $510,559$.

34. Dendronephthya (Roxasia) punctata KÜKENTHAL 560,564 , 569 (?).

35. Dendronephthya (Roxasia) mollis (HoLM) $652,797$.

36. Dendronephthya (Morchellana) castanea UTINOMI 775.

37. Dendronephthya (Morchellana) acaulis KüKENTHAL $495,754,819$.

38. Dendronephthya (Morchellana) querciformis KÜKENTHAL 552.

39. Dendronephthya (Morchellana) habereri KüKENTHAL 66, 404, 474, $476,535,536$.

40. Dendronephthya (Morchellana) pumilio (STUDER) 62.

41. Dendronephthya spp. undet. $685,686,692$.

42. Stereonephthya japonica UTINomI $509,642,650$, $656,657,671$, $675,677,682$ 696.

43. Coronephthya macrospiculata (Thomson \& MAckinnon) (n. gen.). 57, 702, 783. Family Nidaliidae

44. Siphonogorgia dofleini KÜKENTHAL $63,96,464$, $708,767$.

45. Siphonogorgia dipsacea (WRIGHT \& STUDER) 700 .

\section{GORGONACEA}

Suborder SCLERAXONIA

Family Paragorgiidae

46. Paragorgia tenuis KInoshita? 792.

\section{Suborder HolaxONIA}

Family Acanthogorgiidae

47. Acanthogorgia multispina KÜKenthaL \& GoRZAwsKY $606,690(?)$.

\section{Family Paramuriceidae}

48. Muricella nitida (VERRILL) 718.

49. Muricella brunnea KÜKENTHAL ? 761.

50. Echinomuricea simplex Uтімомі (n. sp.) 762.

51. Discogorgia hadrospiculata Uтіломі (n. sp.) 779. 


\section{Family Plexauridae}

52. Euplexaura erecta KÜKENTHAL ..................................... 758.

53. Euplexaura attenuata (NuTTING) .................................... 716.

\section{Family Ellisellidae}

54. Verrucella sp. 709.

\section{Family Primnoidae}

55. Plumarella spinosa KInoshitA $687,693(?)$.

\section{PENNATULACEA}

Suborder SESSILIFLORAE

Family Kophobelemnidae

56. Sclerobelemnon burgeri (HERKLOTS) 724.

\section{Family Protoptilidae}

57. Protoptilum orientale NutTing 734.

\section{Family Scleroptilidae}

58. Calibelemnon indicum (ThOMson \& Henderson) 707.

\section{Suborder SUBSELLIFLORAE \\ Family Virgulariidae}

59. Scytalium splendens (Thomson \& Henderson) $651,723,729$.

\section{Family Pennatulidae}

60. Pennatula phosphorea var. longispinosa MorofF 784.

61. Pennatula murrayi KöLliKeR $176(?), 703$.

62. Pennatula naresi KöLliKer 712.

63. Leioptilus fimbriatus (HERKLOTs) 769. 